\title{
Letter regarding "Effect of laser on pain relief and wound healing of recurrent aphthous stomatitis: a systematic review"
}

\author{
Nasrin Zand ${ }^{1}$ (D) Afshan Shirkavand ${ }^{2}$
}

Received: 3 June 2017 / Accepted: 31 July 2017 / Published online: 12 August 2017

(C) Springer-Verlag London Ltd. 2017

We studied with great interest the article entitled, "Effect of laser on pain relief and wound healing of recurrent aphthous stomatitis: a systematic review" authored by Suter and colleagues [1]. In this valuable systematic review, the authors evaluated the potential benefits of laser application in management of recurrent aphthous stomatitis. We would like to give explanation regarding the above paper published recently.

In Table 2 of Dr. Valerie G. A. Suter's paper, the total energy used in NTCLT has been stated to be $5-10 \mathrm{~J}$; however, the energy delivered to the surface of the lesions is much lower than the figures above.

For application of $\mathrm{CO} 2$ laser as a non-thermal laser for photobiomodulation, some measures should be considered [2-5]:

- Irradiation of the lesion through a thick layer of transparent gel with high water content

- Application of the CO2 laser with defocused handpiece, 5-6 $\mathrm{mm}$ distant from the surface of the lesion to make the beam wide enough

- Rapid scanning of the lesion with circular motion of the handpiece

Nasrin Zand

zand_nas@yahoo.com

1 Medical Laser Research Center (MLRC), Academic Center for Education, Culture and Research (ACECR), Tehran, Iran

2 Laser and Plasma research center, Shahid Beheshti University; Medical Laser Research Center (MLRC), Academic Center for Education, Culture and Research (ACECR), Tehran, Iran
With these considerations, $\mathrm{CO} 2$ laser can be applied as a photobiomodulative laser. This technique which was initially termed NACLT, after indicating its non-thermal nature, called Non-Thermal CO2 Laser Therapy (NTCLT) [5] to avoid misinterpretation with high power fractional non-ablative $\mathrm{CO} 2$ lasers used for skin rejuvenation.

The results of thermometry revealed that final power output of $\mathrm{CO} 2$ laser beam after irradiation through the gel decreased significantly to the range of milliwatts [2]. Hence, the real energy delivered to the surface of the lesion in NTCLT is in the range of millijules. We hope we will be able to report the results of our recent study about the physical aspects of NTCLT with more accurate information about the real energy applied at the surface of the lesion in NTCLT soon.

\section{References}

1. Suter VGA, Sjölund S, Bornstein MM (2017) Effect of laser on pain relief and wound healing of recurrent aphthous stomatitis: a systematic review. Lasers Med Sci 32:953-963

2. Zand N, Ataie-Fashtami L, Djavid GE et al (2009) Relieving pain in minor aphthous stomatitis by a single session of non-thermal carbon dioxide laser irradiation. Lasers Med Sci 24(4):515-520

3. Zand N, Ataie-Fashtami L, Fateh M, Djavid GE, Fatemi S, Shirkavand A (2012) Promoting wound healing in minor recurrent aphthous stomatitis by non-thermal, non-ablative $\mathrm{CO} 2$ laser therapy. Photomed Laser Surg 30(12):719-723

4. Zand N (2012) Non-thermal, non-ablative CO2 laser therapy (NACLT): a new approach to relieve pain in some painful oral diseases. In: Dumitras DC (ed) CO2 laser-optimization and application. InTech-Open Access publisher, Rijeka, p 387-414

5. Zand N, Mansouri P, Fateh M, Ataie-Fashtami L et al (2017) Relieving pain in oral lesions of pemphigus vulgaris using the nonablative, non-thermal, CO2 laser therapy (NTCLT): preliminary results of a novel approach. J Lasers Med Sci 8(1):7-12 